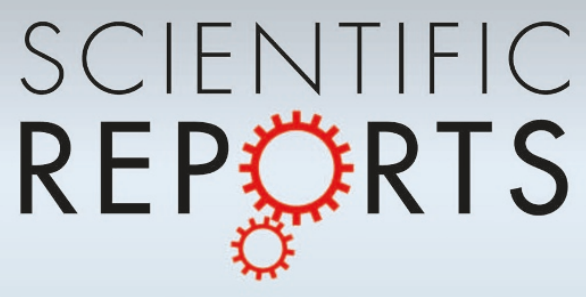

OPEN

SUBJECT AREAS:

SUSTAINABILITY

PHOTOCATALYSIS

SYNTHESIS AND PROCESSING

POROUS MATERIALS

Received

13 May 2013

Accepted

21 June 2013

Published

8 July 2013

Correspondence and requests for materials should be addressed to Y.Z. (Yuanjian. Zhang@seu.edu.cn) or Z.S. (z.schnepp@ bham.ac.uk)

\section{Biopolymer-Activated Graphitic Carbon Nitride towards a Sustainable Photocathode Material}

\author{
Yuanjian Zhang' ', Zoë Schnepp ${ }^{2}$, Junyu Cao², Shuxin Ouyang' ${ }^{2}$, Ying Li' ${ }^{1}$ Jinhua Ye² \& Songqin Liu'
}

'School of Chemistry and Chemical Engineering, Southeast University, Nanjing 211189 , China, ${ }^{2}$ International Center for Young Scientists (ICYS), Center for Materials Nanoarchitectonics (MANA), Research Unit for Environmental Remediation Materials, National Institute for Materials Science (NIMS), 1-1 Namiki, Tsukuba, 305-0044, Japan.

Photoelectrochemical (PEC) conversion of solar light into chemical fuels is one of the most promising solutions to the challenge of sustainable energy. Graphitic carbon (IV) nitride polymer (g-CN) is an interesting sustainable photocathode material due to low-cost, visible-light sensitivity, and chemical stability up to $500^{\circ} \mathrm{C}$ in air. However, grain boundary effects and limited active sites greatly hamper g-CN activity. Here, we demonstrate biopolymer-activation of g-CN through simultaneous soft-templating of a sponge-like structure and incorporation of active carbon-dopant sites. This facile approach results in an almost $300 \%$ increase in the cathodic PEC activity of g-CN under simulated solar-irradiation.

ustainable energy is one of the biggest challenges facing our current society. One solution is to use photoelectrochemical (PEC) cells incorporating semiconductors to directly convert solar energy into chemical fuels $^{1,2}$. As synthesized graphitic carbon (IV) nitride polymer (g-CN, Fig. 1) is an attractive metal-free semiconductor that can be used in photocatalytic water splitting and dye degradation and optoelectronic conversion under visible light without photo-corrosion, and also catalysis of various other important reactions ${ }^{3-11}$. In general, g-CN is a low-cost material with a band gap of approximately $2.7 \mathrm{eV}$, depending on structural variations or the presence of adatoms ${ }^{12-15}$. More interestingly, due to the graphitic structure, g-CN has a remarkably high stability against oxidation in air up to $500^{\circ} \mathrm{C}^{4}$, which is superior to most other organic counterparts $\left(\sim 150^{\circ} \mathrm{C}\right)^{16}$.

One challenge for g-CN is that the conversion efficiency of pristine g-CN solids in sunlight is low, due to limited visible light absorption, low surface area and grain boundary effects. For optimizing light absorption, there has been intensive investigation of various ionic, covalent and non-covalent chemical dopants ${ }^{12-15,17-21}$. Reducing the grain size is in principle a general solution for improving electron transport due to high overall surface area, but is still difficult for g-CN. For instance, hard templating strategies using mesoporous silica, silica nanoparticles and anodic aluminum oxide (AAO) membrane have been used to prepare nanostructured $\mathrm{g}-\mathrm{CN}^{22-27}$. However, these require hazardous etchants such as $\mathrm{HF}$ as well as being time consuming, multi-step procedures.

Here, we demonstrate a sustainable one-pot route to sponge-like structures of g-CN with active carbon-doped sites. This method exploits the inherent self-organization of biopolymers such as alginate (seaweed polysaccharide) and gelatin (polypeptide) as an in situ template (see Fig. 1). Many biopolymers readily form extended hierarchical structures in water through e.g. coiled coils and double or triple helices. They also exhibit diverse chemical functionality (e.g. carboxylate, hydroxyl) and thus strongly bind to metal cations forming gels with high thermal stability. These factors have enabled them to act as an efficient template for nanostructures of diverse systems such as metal oxides and carbides ${ }^{28-36}$. To date, however, there are few examples of using biopolymers as 'soft' templates for 'soft' (organic) nanomaterials. In this work, we use abundant biopolymers as a template to disperse and bind a common carbon nitride precursor. The resulting sponge-like g-CN nanostructure, the simultaneous carbon doping, and their effects on the activity as a photocathode material (a proof of concept application of semiconducting $\mathrm{g}-\mathrm{CN}$ ) is presented.

\title{
Results
}

Aqueous dicyandiamide (DCDA, the precursor for g-CN) was mixed with alginate or gelatin solution and then dried to give solids with 5 or $10 \%$ biopolymer by mass. These were heated to $550^{\circ} \mathrm{C}$ in air for 4 hours with a 4 hour ramp time and the as-prepared samples were named as Alg- $\mathrm{x}-\mathrm{CN}$ or $\mathrm{Gel}-\mathrm{x}-\mathrm{CN}, \mathrm{x}=5$ or 10 . As a control, pristine g-CN was also prepared using only DCDA under the same conditions. The structure and texture of these carbon 


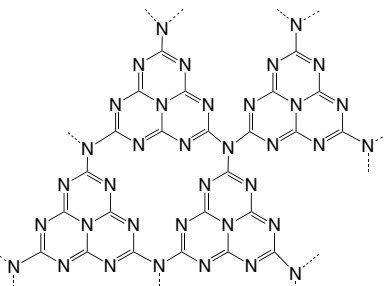

g-CN network

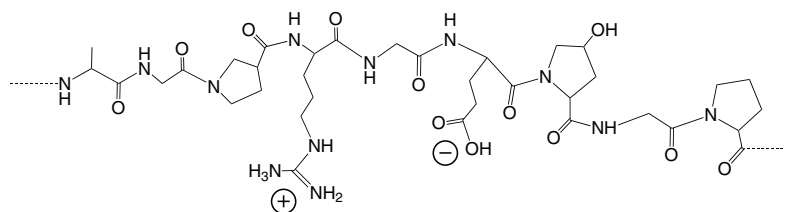

Gelation

Figure 1 Ideal molecular structures of graphitic carbon nitride (the defects are not shown for clarity), alginate, and gelatin.

nitride polymers were first evaluated by Fourier transform infrared spectra (FT-IR) and X-ray diffraction (XRD). It was found that all FT-IR spectra of Alg-X-CN and Gel-x-CN are very similar to g-CN, showing typical stretches of the $\mathrm{C}-\mathrm{N}$ extended network ranging from 800 to $1800 \mathrm{~cm}^{-1}$ (Supplementary Fig. S1). Furthermore, the XRD patterns of the Alg-x-CN and Gel-x-CN maintain the same characteristic (002) interlayer-stacking peak as the control g-CN (Supplementary Fig. S2). Thus, the original graphitic C-N motif of $\mathrm{Alg}-\mathrm{x}-\mathrm{CN}$ and Gel-x-CN stayed mostly unchanged with respect to the pristine $\mathrm{g}-\mathrm{CN}$.

In contrast, the morphology and microstructure of the carbon nitride is dramatically changed upon interaction with the biopolymers during calcination. Scanning and transmission electron microscopy (SEM and TEM) images in Fig. 2 and Fig. S3 show that the control g-CN solid was a monolith, while Alg-x-CN, and Gel-x-CN became sponge-like. Accordingly, the $\mathrm{N}_{2}$ adsorption/desorption isotherms (Supplementary Fig. S4) show abrupt uptake at $\mathrm{P} / \mathrm{P}_{0}>0.9$ due to the inter particle voids. Moreover, when the ratio of biopolymers to dicyandiamide further increased, some wrinkled grapheneliked sheets were also observed (e.g. Alg-10-CN, see Supplementary Fig. S3a inset). This resulted in a substantial increase in BET surface area for all of the biopolymer-activated samples (Supplementary Fig. S4 and Table S1). For example, the surface area of Alg-5-CN (42 $\mathrm{m}^{2} /$ g) was 4 times that of control g-CN $\left(10 \mathrm{~m}^{2} / \mathrm{g}\right)$, and that of Alg-10-CN $\left(63 \mathrm{~m}^{2} / \mathrm{g}\right)$ was even higher (ca. 6 times).

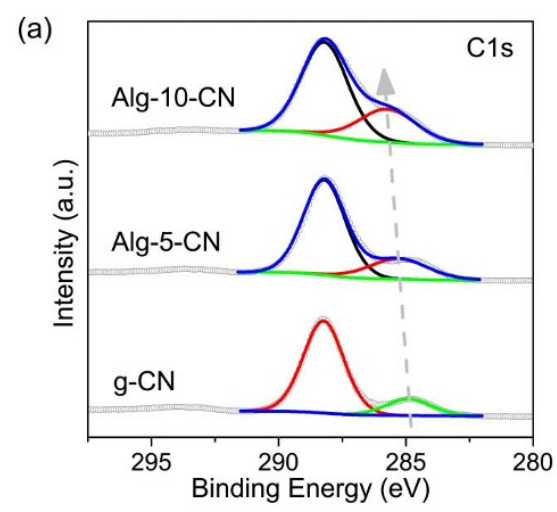

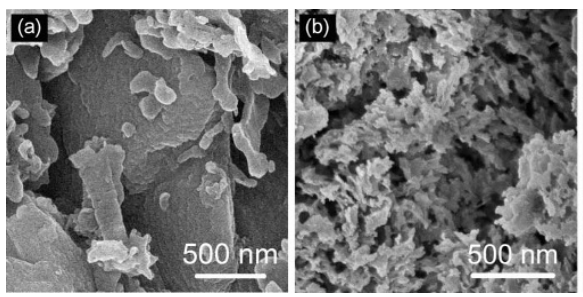

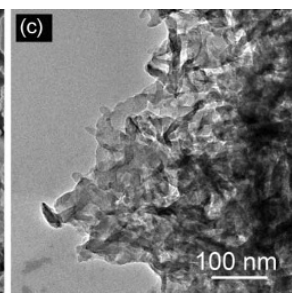

Figure $2 \mid$ Microstructure of carbon nitrides. SEM images of pristine g$\mathrm{CN}$ (a) and Alg-5-CN (b), and TEM image of Alg-5-CN (c).

This result is particularly interesting since the preparation of "soft" nanomaterials by so-called 'soft-templating' still presents a considerable challenge ${ }^{37}$. For example, it was reported that preparation of nanostructured g-CN using surfactants such as cetyltrimethylammonium bromide (CTAB), sodium dodecyl sulfate (SDS), Triton $\mathrm{X}-100, \mathrm{P} 123$, and F127 under the same condition was difficult, unless under a sophisticated calcination-program and/or with additional pretreatment for the precursors ${ }^{38,39}$. The practical problem was that condensation towards larger extended $\mathrm{CN}$-structures takes place $\left(\sim 390^{\circ} \mathrm{C}\right)$ above the complete decomposition temperature of the soft-templates. In this regard, alginate and gelatin provide a unique alternative, since they combine self-assembly characteristics analogous to surfactants with relatively high thermal stability. This is supported by thermogravimetric (TG) curves which reveal that a significant amount of carbonized alginate (20wt\%) and gelatin (48 wt\%) existed up to $390^{\circ} \mathrm{C}$, acting as porogens in the formation of the g-CN macrostructure (Supplementary Fig. S5).

It was noted that when the ratio of alginate or gelatin to DCDA increased, the $\mathrm{C} / \mathrm{N}$ ratio of the as-prepared Alg-x-CN and Gel-x-CN increased slightly in comparison with g-CN (Supplementary Table S1). X-ray photoelectron spectroscopy (XPS) was used to obtain further information about these carbon dopants (Fig. 3a and Supplementary Fig. S6). As expected, one predominant C1s peak with a binding energy of $288.2 \mathrm{eV}$ is observed for all carbon nitride samples, corresponding to the typical aromatic $\mathrm{C}-\mathrm{N}-\mathrm{C}$ coordination in a graphitic carbon nitride framework ${ }^{3}$. Importantly, a minor peak was also noted, which shifts upward from ca. $284 \mathrm{eV}$ to $286 \mathrm{eV}$ as the $\mathrm{C} / \mathrm{N}$ ratio increases. This indicates the formation of some new carbon species, presumably from replacement of nitrogen in the conjugated framework of graphitic carbon nitride, since the polysaccharide and polypeptides would be fully decomposed from the calcination step and the unique high thermal stability of the g-CN was almost unchanged (Supplementary Fig. S7).

The incorporation of carbon-dopants into the carbon nitride polymer has a remarkable effect on the electronic properties. The electronic spin resonance (ESR) spectra at $4.2 \mathrm{~K}$ shows a major signal

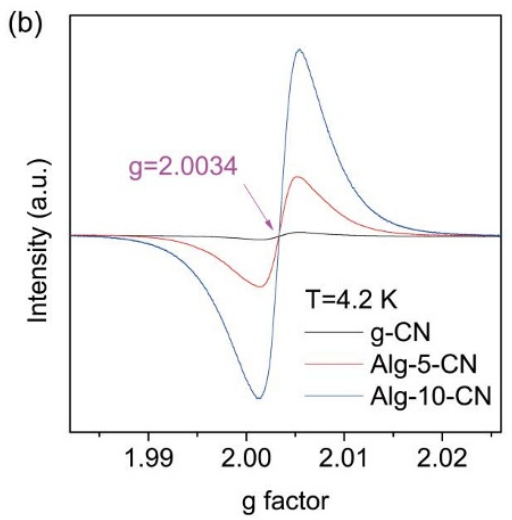

Figure 3 Dopants in carbon nitrides. C1s XPS (a) and ESR spectra (b) of g-CN, Alg-5-CN, and Alg-10-CN. 

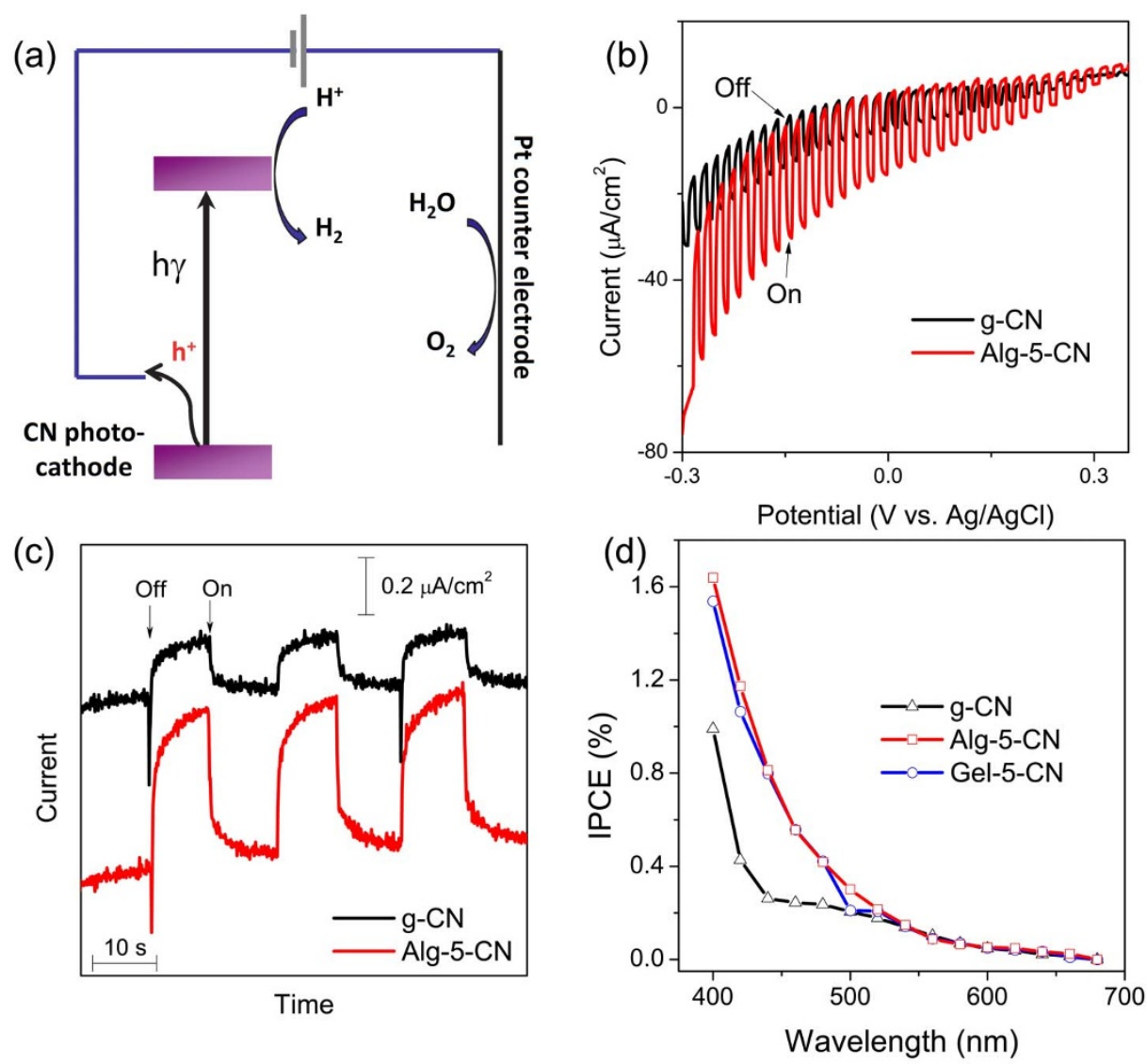

Figure $4 \mid$ Photoelectrochemical (PEC) properties of carbon nitrides in $0.1 \mathrm{M} \mathrm{KCl}$ aqueous solution. (a) The PEC cell configuration and possible charge transfer (the reference electrode was omitted for clarity). (b) Characteristic photocurrents at g-CN (black) and Alg-5-CN (red) photocathode under a chopped sunlight simulator at a scan rate of $10 \mathrm{mV} / \mathrm{s}$. (c) Transient current response of g-CN (black) and Alg-5-CN (red) at -0.2 V vs Ag/AgCl (sat. $\mathrm{KCl}$ ) under chopped monochromatic light at $440 \mathrm{~nm}$. (d) Photo action spectra of g-CN (black), Alg-5-CN (red), and Gel-5-CN (blue) biased at $-0.2 \mathrm{~V}$ vs Ag/AgCl (sat. $\mathrm{KCl})$.

where $\mathrm{g}=2.0034$ (characteristic of paramagnetic aromatic carbon atoms) for all the samples, which increases in intensity with increasing carbon content (Fig. 3b). This clearly suggests the electronic integration of new carbon species with unpaired electrons in the lattice of the graphitic carbon nitride, which is significant for potential applications since certain amount of dopants favor efficient charge separation. Moreover, the corresponding optical absorption peaks from these carbon nitrides were shifted to lower energies with increasing carbon content (Supplementary Fig. S8a), indicating the extension of the $\pi$-conjugated structure from these carbon dopants. It was also noted that the intensity of the photoemission was strongly suppressed (Supplementary Fig. S8b), which is an essential indicator for effective separation and collection of excited electrons and holes.

\section{Discussion}

In order to investigate the potential of these materials as photocathode materials, the PEC measurements were performed using a three-electrode configuration (Fig. 4a) in an aqueous solution containing $0.1 \mathrm{M} \mathrm{KCl}$. It was observed that the cathodic photoresponses of g-CN and Alg-5-CN gradually increased when the biased potential became more negative (Fig. $4 \mathrm{~b}$ ), and the photocurrents were prompt, steady, and reproducible during repeated on/off cycles (Fig. 4c). More interestingly, the substantial photocurrent of Alg-5-CN was much larger than that of g-CN under all biased potentials, strongly indicating the positive impact of the biopolymer-activation. For example, when the bias potential was ca. $-0.2 \mathrm{~V}$, the anodic photocurrent of Alg-5-CN was 2.6 times of that of g-CN in the simulated sunlight. Gel-5-CN showed a similarly enhanced photocurrent.
The sponge-like nanostructures of the activated carbon nitride should account in part for the improved photo-response. In general, compared with the monolith, the sponge-like nanostructure would shorten the diffusion path of free carriers, enhance mass transfer during the reaction, provide more active sites, and enhance the optical absorption of photoelectrodes as well via multiple internal reflections (see intrinsic band-absorption up to $500 \mathrm{~nm}$, Fig. S8a). Interestingly, however, Alg-10-CN and Gel-10-CN, which had the highest surface area, showed a lower PEC activity (Supplementary Fig. S9). Thus, a low ratio of the biopolymers to DCDA (i.e. 5\%) seemed favorable for creating sponge-like carbon nitride solids with higher PEC activity. As shown in Supplementary Table S1, indeed, when the ratio of the biopolymers to DCDA changed, not only the morphology (roughly indicated by the surface area) but also the concentration of dopants varied. Both porosity and dopants should be helpful for the enhanced photocurrents, but it is still difficult to quantify their contribution currently. For example, the BET surface area of Alg-5-CN (42 m²/g) is larger than that of Gel-5-CN $\left(34 \mathrm{~m}^{2} /\right.$ g), however, they had a very similar photocurrent activity (see Fig. 4d), which was presumably due to the fact that the concentration of carbon dopants for Gel-5-CN (C/N 1.17) is higher than that of Alg-5-CN (C/N 1.08). Besides, it is recognized that an appropriate concentration of dopants in semiconductors can improve the mobility of the charge-carriers, but too high a concentration can increase unwanted charge recombination thus resulting in a reduced activity. Therefore, a more detailed screening of the optimum concentration of the biopolymers is required for further improvement of this system. 
The incident photon-to-charge conversion efficiencies (IPCEs), biased at $-0.2 \mathrm{~V}$ of g-CN, Alg-5-CN and Gel-5-CN as a function of wavelength, were also measured and are shown in Figure $4 \mathrm{~d}$. Interestingly, the IPCEs of Alg-5-CN and Gel-5-CN were much higher than that of g-CN under the monochromatic irradiation up to $500 \mathrm{~nm}$ (e.g. 3.1 times at $440 \mathrm{~nm}$ ), while they were essentially the same from above $550 \mathrm{~nm}$. This is consistent with the photoresponse data under solar simulation. Moreover, it suggests that the extended optical absorption of Alg/Gel-5-CN by carbon dopants (Fig. S8a) did not contribute to IPCEs, probably due to the weakened redox ability during the band-gap shrinkage ${ }^{21}$. Thus, the photo-responses here were derived from the intrinsic band excitation of carbon nitride in all cases. More notably, the highest IPCE measured $(1.2 \%$ at $\lambda$ $=420 \mathrm{~nm}$ ) is comparable to those in existing reports of mesoporous polymeric carbon nitrides by hard-templating ${ }^{5,27}$ and even some $\mathrm{p}$ type metal-containing counterparts (e.g. $\mathrm{Cu}-\mathrm{Ti}-\mathrm{O})^{40}$. While this is still lower than those of state-of-the-art photocathodes (e.g. $40 \%$ for sophisticated $\left.\mathrm{Cu}_{2} \mathrm{O} / \mathrm{ZnO}: \mathrm{Al} / \mathrm{TiO}_{2} / \mathrm{Pt}\right)^{41}$, this promising preliminary result suggests that there is considerable scope for improvement in either photoelectrode layout ${ }^{41}$, co-catalyst ${ }^{5,27}$, or both. Furthermore, n-typed semiconductors such as $\mathrm{TiO}_{2}, \mathrm{ZnO}, \mathrm{Fe}_{2} \mathrm{O}_{3}$ and $\mathrm{WO}_{3}$ have been intensively investigated as photoanodes for PEC water oxidation $^{1,42-47}$. In contrast, the p-typed semiconductors, especially polymeric ones, which could be used as photocathodes for PEC water reduction are poorly represented ${ }^{48}$. In this regard, it is also interesting to consider g-CN as a photocathode material for solar fuels.

Nevertheless, the BET area values developed by soft-templating here were not as high as those by hard-templating ${ }^{27}$. However, it should be aware that the BET area is not the higher, the better for photo(electro)catalytic applications. For example, Wang et al prepared the mesoporous g-CN with BET area from 67 to $373 \mathrm{~m}^{2} / \mathrm{g}$ using silica as the hard template, but found that the sample with BET area of $67 \mathrm{~m}^{2} / \mathrm{g}$ had the highest activity ${ }^{27}$. This phenomenon was also observed in this study (Fig. 4 b and Supplementary S9). It is generally because when the surface area increases, the defects where the excitions recombine also increase.

In summary, we have shown that alginate and gelatin are two promising activating agents for semiconducting graphitic carbon nitride solids. Synergistic interactions between the carbon nitride precursor and the self-organized biopolymer precursor create a sponge-like porosity with an enhanced active sites. Furthermore, by balancing the biopolymer content, a favorable concentration of active carbon dopant sites can be created in the graphitic carbon nitride network to enhance charge mobility. As a result, this proofof-concept application shows the photocathodic performance of carbon nitrides after the activation in the best case could be greatly improved by a factor of nearly $300 \%$ under solar irradiation. Thanks to the flexible variability of molecular structures (e.g. hyaluronan, cellulose, etc.), physicochemical properties, and other functionalities (e.g. sulfonate-rich carrageenan which could be used for sulfur doping) of biopolymers, it is highly feasible to further increase the PEC activity by better balancing electronic structures (e.g. bandgap and redox ability), change-carrier mobility and surface area. Besides the facile synthesis, the abundance of both starting materials will no doubt also be beneficial to push the activated carbon nitrides as sustainable metal-free photocathode materials e.g. for PEC hydrogen evolution from water. Work focused on the more detailed activation mechanism (e.g. relationship between catalytic activity and amount of carbon dopants) and optimizing the photoelectrode layout and co-catalysts of carbon nitride solids for higher efficiency is ongoing.

\section{Methods}

Preparation of biopolymer-activated graphitic carbon nitride. Aqueous dicyandiamide (DCDA, 99\%) was mixed with ammonium alginate (Protacid F20NM, FMC biopolymer, prepared by dispersing alginic acid in water and adjusting the $\mathrm{pH}$ to 7 with $\mathrm{NH}_{4} \mathrm{OH}$ ) or gelatin (Type A, from porcine skin, Sigma-Aldrich, G2500, prepared by dissolving in hot water) solution and then dried to give solids with 5 or $10 \%$ biopolymer by mass. The samples were heated to $550^{\circ} \mathrm{C}$ in air for 4 hours with a 4 hour ramp time and the as-prepared samples were named as Alg-x-CN or Gel-x-CN, $\mathrm{x}=5$ or 10 . As the control, pristine graphitic carbon nitride $(\mathrm{g}-\mathrm{CN})$ was also prepared using only DCDA under the same condition.

Photoelectrochemical experiments were performed in a conventional threeelectrode cell with a platinum wire as the auxiliary electrode and an $\mathrm{Ag} / \mathrm{AgCl}$ (saturated $\mathrm{KCl}$ ) as the reference electrode on a $\mathrm{CHI}$ 650A workstation (USA). A solar simulator (AM 1.5G, Peccell, Japan) was used as the light source. The light energy density in the vicinity of the photoelectrodes was measured by a USHIO energy meter (USR-40D-B, Japan) to be ca. $84 \mathrm{~mW} / \mathrm{cm}^{2}(200-800 \mathrm{~nm})$. The photoelectrode (working electrode, $0.49 \mathrm{~cm}^{2}$ ) was inserted in a $0.1 \mathrm{M} \mathrm{KCl}$ aqueous solution with the glass side facing the incident light (to eliminate the influence from the slight variation of film-thickness). The photoelectrode was prepared by spreading aqueous slurries of various carbon nitride solids on ITO glass $\left(10 \mathrm{ohm} / \mathrm{cm}^{2}\right)$ with a glass rod, using adhesive tapes as spaces. It was dried in air and then annealed at $150^{\circ} \mathrm{C}$ for $10 \mathrm{~min}$. The suspension was prepared by grinding $5 \mathrm{mg}$ of carbon nitride solids, $10 \mu \mathrm{L}$ of PEDOT-PSS (Sigma-Aldrich, 1.3-1.7\%) aqueous solution and $50 \mu \mathrm{L}$ of water. For incident photon-to-electron conversion efficiency (IPCE) measurement, the monochromatic light was generated by a $500 \mathrm{~W}$ Xe lamp (USHIO Optical Modulex X500, Japan) with a monochromator (M10, JASCO, Japan), and the energy of the monochromatic light was measured by USHIO energy meter. The IPCE was calculated by normalizing the photocurrent values for incident light energy and intensity by use of the equation, i.e., $\operatorname{IPCE}(\%)=100 * 1240 * \mathrm{I}_{\mathrm{pc}} /\left(\mathrm{I}_{\mathrm{inc}} * \lambda\right)$ where $\mathrm{I}_{\mathrm{pc}}$ is photocurrent $\left(\mathrm{A} / \mathrm{cm}^{2}\right), \mathrm{I}_{\mathrm{inc}}$ is the incident light intensity $\left(\mathrm{W} / \mathrm{cm}^{2}\right)$ and $\lambda$ is the wavelength $(\mathrm{nm})$

Characterization. Fourier transform infrared spectra (FT-IR) spectra were collected with a Nicolet 4700 FTIR spectrometer (Thermo, USA), equipped with an attenuated total reflection (ATR) setup. X-ray diffraction (XRD) patterns were recorded on a RINT-ultra3 diffractometer ( $\mathrm{Cu}$ K $\alpha$ radiation, Rigaku, Japan). The SEM images were measured on a Hatachi SU-8000 (2 kV, Japan). TEM images were obtained on a JEM2100F (200 kV, Japan). UV-vis absorption spectra were recorded on a Shimadzu UV-2500PC UV-vis spectrometer with a diffuse reflectance accessory, and $\mathrm{BaSO}_{4}$ was used as the reference sample (100\% reflectance). X-ray Photoelectron Spectroscopy (XPS) experiments were performed in a Theta probe (Thermo Fisher) using monochromated $\mathrm{Al} \mathrm{K} \alpha \mathrm{X}$-rays at hv $=1486.6 \mathrm{eV}$. TGA measurements were performed on SII Exstar 6000 (TG-DTA 6200) in air $\left(50 \mathrm{~mL} / \mathrm{min}, 10^{\circ} \mathrm{C} / \mathrm{min}\right)$. The Brunauer-Emmett-Teller (BET) surface area was obtained by nitrogen sorption experiments using a Quantachrome Autosorb Automated Gas Sorption System at $77 \mathrm{~K}$. Elemental analysis was undertaken by Carbon/Sulfur Determinator (LECO Co., CS-444LS) that equips with induction furnace, infrared absorption detector, and uses $\mathrm{CaCO}_{3}$ as the standard sample, and Oxygen/Nitrogen Determinator (LECO Co., TC-436AR) that equips with impulse furnace and thermal conductivity detector, and $\mathrm{Si}_{3} \mathrm{~N}_{4}$ as the standard sample. Electronic spin resonance (ESR) spectra were obtained on JEOL JES-FA200 Electron Spin Resonance Spectrometer at $4.2 \mathrm{~K}$

1. Fujishima, A. \& Honda, K. Electrochemical Photolysis of Water at a Semiconductor Electrode. Nature 238, 37-38 (1972)

2. Grätzel, M. Photoelectrochemical cells. Nature 414, 338-344 (2001).

3. Thomas, A. et al. Graphitic carbon nitride materials: variation of structure and morphology and their use as metal-free catalysts. J. Mater. Chem. 18, 4893-4908 (2008).

4. Zhang, Y., Mori, T. \& Ye, J. Polymeric Carbon Nitrides: Semiconducting Properties and Emerging Applications in Photocatalysis and Photoelectrochemical Energy Conversion. Sci. Adv. Mater. 4, 282-291 (2012).

5. Wang, X. C. et al. A metal-free polymeric photocatalyst for hydrogen production from water under visible light. Nature Mater. 8, 76-80 (2009).

6. Yan, S. C., Li, Z. S. \& Zou, Z. G. Photodegradation Performance of g- $\mathrm{C}_{3} \mathrm{~N}_{4}$ Fabricated by Directly Heating Melamine. Langmuir 25, 10397-10401 (2009).

7. Zhang, Y. J. \& Antonietti, M. Photocurrent Generation by Polymeric Carbon Nitride Solids: An Initial Step towards a Novel Photovoltaic System. Chem.-Asian J. 5, 1307-1311 (2010).

8. Datta, K. K. R., Reddy, B. V. S., Ariga, K. \& Vinu, A. Gold Nanoparticles Embedded in Mesoporous Carbon Nitride Stabilizer for Highly Efficient ThreeComponent Coupling Reaction. Angew. Chem. Int. Ed. 49, 5961-5965 (2010).

9. Zheng, Y.et al. Nanoporous Graphitic- $\mathrm{C}_{3} \mathrm{~N}_{4} @$ Carbon Metal-Free Electrocatalysts for Highly Efficient Oxygen Reduction. J. Am. Chem. Soc. 133, 20116-20119 (2011).

10. Xiang, Q., Yu, J. \& Jaroniec, M. Preparation and Enhanced Visible-Light Photocatalytic $\mathrm{H}_{2}$-Production Activity of Graphene/ $\mathrm{C}_{3} \mathrm{~N}_{4}$ Composites. J. Phys. Chem. C 115, 7355-7363 (2011).

11. Wirnhier, E. et al. Poly(triazine imide) with Intercalation of Lithium and Chloride Ions $\left[\left(\mathrm{C}_{3} \mathrm{~N}_{3}\right)_{2}\left(\mathrm{NH}_{\mathrm{x}} \mathrm{Li}_{1-\mathrm{x}}\right)_{3} \cdot \mathrm{LiCl}\right]$ : A Crystalline 2D Carbon Nitride Network. Chem. Eur. J. 17, 3213-3221 (2011).

12. Zhang, Y. J., Thomas, A., Antonietti, M. \& Wang, X. C. Activation of carbon nitride solids by protonation: Morphology changes, enhanced ionic conductivity, and photoconduction experiments. J. Am. Chem. Soc. 131, 50-51 (2009).

13. Liu, G. et al. Unique Electronic Structure Induced High Photoreactivity of SulfurDoped Graphitic $\mathrm{C}_{3} \mathrm{~N}_{4}$. J. Am. Chem. Soc. 132, 11642-11648 (2010). 
14. Zhang, Y. J., Mori, T., Ye, J. H. \& Antonietti, M. Phosphorus-Doped Carbon Nitride Solid: Enhanced Electrical Conductivity and Photocurrent Generation. J. Am. Chem. Soc. 132, 6294-6295 (2010)

15. Wang, Y., Li, H., Yao, J., Wang, X. \& Antonietti, M. Synthesis of boron doped polymeric carbon nitride solids and their use as metal-free catalysts for aliphatic C-H bond oxidation. Chem. Sci. 2, 446 (2011).

16. Cho, S. et al. A Thermally Stable Semiconducting Polymer. Adv. Mater. 22, 1253 1257 (2010).

17. Guo, Y., Chu, S., Yan, S., Wang, Y. \& Zou, Z. Developing a polymeric semiconductor photocatalyst with visible light response. Chem. Commun. 46, 7325 (2010).

18. Yan, S. C., Li, Z. S. \& Zou, Z. G. Photodegradation of Rhodamine B and Methyl Orange over Boron-Doped g- $\mathrm{C}_{3} \mathrm{~N}_{4}$ under Visible Light Irradiation. Langmuir 26, 3894-3901 (2010)

19. Wang, Y. et al. Excellent Visible-Light Photocatalysis of Fluorinated Polymeric Carbon Nitride Solids. Chem. Mater. 22, 5119-5121 (2010).

20. Zhang, Y., Mori, T., Niu, L. \& Ye, J. Non-covalent doping of graphitic carbon nitride polymer with graphene: controlled electronic structure and enhanced optoelectronic conversion. Energy Environ. Sci. 4, 4517 (2011).

21. Zhang, J. et al. Co-Monomer Control of Carbon Nitride Semiconductors to Optimize Hydrogen Evolution with Visible Light. Angew. Chem. Int. Ed. 51, 3183-3187 (2012).

22. Vinu, A. Two-Dimensional Hexagonally-Ordered Mesoporous Carbon Nitrides with Tunable Pore Diameter, Surface Area and Nitrogen Content. Adv. Funct. Mater. 18, 816-827 (2008).

23. Lee, E. Z., Jun, Y.-S., Hong, W. H., Thomas, A. \& Jin, M. M. Cubic Mesoporous Graphitic Carbon(IV) Nitride: An All-in-One Chemosensor for Selective Optical Sensing of Metal Ions. Angew. Chem. Int. Ed. 49, 9706-9710 (2010).

24. Goettmann, F., Fischer, A., Antonietti, M. \& Thomas, A. Chemical synthesis of mesoporous carbon nitrides using hard templates and their use as a metal-free catalyst for friedel-crafts reaction of benzene. Angew. Chem. Int. Ed. 45, 44674471 (2006).

25. Li, X.-H. et al. Condensed Graphitic Carbon Nitride Nanorods by Nanoconfinement: Promotion of Crystallinity on Photocatalytic Conversion. Chem. Mater. 23, 4344-4348 (2011).

26. Kailasam, K., Epping, J. D., Thomas, A., Losse, S. \& Junge, H. Mesoporous carbon nitride-silica composites by a combined sol-gel/thermal condensation approach and their application as photocatalysts. Energy Environ. Sci. 4, 4668 (2011).

27. Wang, X. C. et al. Polymer Semiconductors for Artificial Photosynthesis: Hydrogen Evolution by Mesoporous Graphitic Carbon Nitride with Visible Light. J. Am. Chem. Soc. 131, 1680-1681 (2009).

28. Schnepp, Z. Biopolymers as a Flexible Resource for Nanochemistry. Angew. Chem. Int. Ed. 52, 1096 (2013).

29. Tingaut, P., Zimmermann, T. \& Sèbe, G. Cellulose nanocrystals and microfibrillated cellulose as building blocks for the design of hierarchical functional materials. J. Mater. Chem. 22, 20105 (2012).

30. Walsh, D., Arcelli, L., Ikoma, T., Tanaka, J. \& Mann, S. Dextran templating for the synthesis of metallic and metal oxide sponges. Nature Mater. 2, 386-390 (2003).

31. Laurent, S. et al. Magnetic Iron Oxide Nanoparticles: Synthesis, Stabilization, Vectorization, Physicochemical Characterizations, and Biological Applications. Chem. Rev. 108, 2064-2110 (2008).

32. Raveendran, P., Fu, J. \& Wallen, S. L. Completely "Green" Synthesis and Stabilization of Metal Nanoparticles. J. Am. Chem. Soc. 125, 13940-13941 (2003).

33. Sarma, T. K. \& Chattopadhyay, A. Starch-Mediated Shape-Selective Synthesis of Au Nanoparticles with Tunable Longitudinal Plasmon Resonance. Langmuir 20, 3520-3524 (2004).

34. Walsh, D., Wimbush, S. C. \& Hall, S. R. Use of the Polysaccharide Dextran as a Morphological Directing Agent in the Synthesis of High-TcSuperconducting $\mathrm{YBa}_{2} \mathrm{Cu}_{3} \mathrm{O}_{7-\delta}$ Sponges with Improved Critical Current Densities. Chem. Mater. 19, 647-649 (2007).

35. Ganesan, R. \& Gedanken, A. Synthesis of $\mathrm{WO}_{3}$ nanoparticles using a biopolymer as a template for electrocatalytic hydrogen evolution. Nanotechnology 19, 025702 (2008).

36. Xia, Y. et al. Biotemplated fabrication of hierarchically porous $\mathrm{NiO} / \mathrm{C}$ composite from lotus pollen grains for lithium-ion batteries. J. Mater. Chem. 22, 9209 (2012).

37. Thomas, A., Goettmann, F. \& Antonietti, M. Hard Templates for Soft Materials: Creating Nanostructured Organic Materials. Chem. Mater. 20, 738-755 (2008).
38. Wang, Y., Wang, X. C., Antonietti, M. \& Zhang, Y. J. Facile One-Pot Synthesis of Nanoporous Carbon Nitride Solids by Using Soft Templates. ChemSusChem 3, 435-439 (2010).

39. Yan, H. Soft-templating synthesis of mesoporous graphitic carbon nitride with enhanced photocatalytic $\mathrm{H}_{2}$ evolution under visible light. Chem. Commun. 48, 3430 (2012).

40. Mor, G. K. et al. p-Type $\mathrm{Cu}-\mathrm{Ti}-\mathrm{O}$ Nanotube Arrays and Their Use in Self-Biased Heterojunction Photoelectrochemical Diodes for Hydrogen Generation. Nano Lett. 8, 1906-1911 (2008).

41. Paracchino, A., Laporte, V., Sivula, K., Grätzel, M. \& Thimsen, E. Highly active oxide photocathode for photoelectrochemical water reduction. Nature Mater. 10, 456-461 (2011)

42. Wolcott, A., Smith, W. A., Kuykendall, T. R., Zhao, Y. \& Zhang, J. Z. Photoelectrochemical Study of Nanostructured ZnO Thin Films for Hydrogen Generation from Water Splitting. Adv. Funct. Mater. 19, 1849-1856 (2009).

43. Xu, M., Da, P., Wu, H., Zhao, D. \& Zheng, G. Controlled Sn-Doping in $\mathrm{TiO}_{2}$ Nanowire Photoanodes with Enhanced Photoelectrochemical Conversion. Nano Lett. 12, 1503-1508 (2012).

44. Zhong, D. K., Sun, J., Inumaru, H. \& Gamelin, D. R. Solar Water Oxidation by Composite Catalyst $/ \alpha-\mathrm{Fe}_{2} \mathrm{O}_{3}$ Photoanodes. J. Am. Chem. Soc. 131, 6086-6087 (2009)

45. Lin, Y., Zhou, S., Sheehan, S. W. \& Wang, D. Nanonet-Based Hematite Heteronanostructures for Efficient Solar Water Splitting. J. Am. Chem. Soc. 133, 2398-2401 (2011).

46. Thimsen, E., Le Formal, F., Grätzel, M. \& Warren, S. C. Influence of Plasmonic Au Nanoparticles on the Photoactivity of $\mathrm{Fe}_{2} \mathrm{O}_{3}$ Electrodes for Water Splitting. Nano Lett. 11, 35-43 (2011)

47. Su, J., Guo, L., Bao, N. \& Grimes, C. A. Nanostructured $\mathrm{WO}_{3} / \mathrm{BiVO}_{4}$ Heterojunction Films for Efficient Photoelectrochemical Water Splitting. Nano Lett. 11, 1928-1933 (2011).

48. Odobel, F., Le Pleux, L. C., Pellegrin, Y. \& Blart, E. New Photovoltaic Devices Based on the Sensitization of p-type Semiconductors: Challenges and Opportunities. Acc. Chem. Res. 43, 1063-1071 (2010).

\section{Acknowledgements}

This work was supported in part by the Fundamental Research Funds for the Central Universities, NSF of Jiangsu province (BK2012317, BK2011591), China, National Natural Science Foundation of China (21203023, 21005016), Grant-in-Aid for Research Activity Start-up (No. KA004) from the Japan Society for the Promotion of Science, and the International Center for Young Scientists, National Institute for Materials Science, Japan. The authors thank FMC Biopolymer for samples of alginate.

\section{Author contributions}

Y.Z. designed the experiment and carried out synthesis of materials and performed SEM/ XPS/ESR/BET/FT-IR/XRD/UV-vis/TGA/elemental analysis. Z.S. performed and interpreted TEM experiments. Y.Z. carried out photoelectrochemical experiments with guidance from J.C., S.O. and Y.L. J.Y. and S.L. provided guidance and discussion. Y.Z. and Z.S. wrote the manuscript. All authors reviewed the manuscript

\section{Additional information}

Supplementary information accompanies this paper at http://www.nature.com/ scientificreports

Competing financial interests: The authors declare no competing financial interests.

How to cite this article: Zhang, Y. et al. Biopolymer-Activated Graphitic Carbon Nitride towards a Sustainable Photocathode Material. Sci. Rep. 3, 2163; DOI:10.1038/srep02163 (2013)

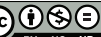

This work is licensed under a Creative Commons AttributionNonCommercial-NoDerivs 3.0 Unported license. To view a copy of this license, visit http://creativecommons.org/licenses/by-nc-nd/3.0 